\title{
VISCOELASTIC SURFACE WAVES ON A STANDARD LINEAR SOLID*
}

\section{BY P. K. CURRIE** (University College, Belfield, Dublin)}

Summary. We investigate the number of surface waves on a standard linear viscoelastic solid. It is shown that two or one surface modes are possible, depending on the frequency and the material parameters. For materials on which two waves are possible, they will generally both be of equal significance over a wide range of frequencies.

1. Introduction. Surface waves propagating over a half-space of viscoelastic material have been investigated recently [1-4]. In the first paper [1] a general analysis showed that (in contrast with elastic materials):

(i) more than one surface wave may be possible;

(ii) the waves may be either retrograde or direct at the top surface;

(iii) the surface wave speed may be greater than the body wave speeds.

In the second paper [2] the number of possible surface waves was investigated numerically as a function of the material parameters, and the results applied to the Kelvin-Voigt and Maxwell models as well as experimental data. The conclusion from the experimental data was that there are typically two surface waves on solid polymers, especially in the transition zone. Detailed experimental data obtained by Waterman [5] for two polymers shows that for one of them only one surface wave is possible at the frequency and temperatures considered [3] but that for the other polymer two surface waves are possible above a certain temperature. This analysis also confirms that it is reasonable to assume incompressibility when examining surface waves on polymers.

The work reported here considers surface waves on an incompressible standard linear solid. This simple model of viscoelastic behavior is more realistic than either the KelvinVoigt or Maxwell models considered in [2]. The results presented here show that over a relatively wide range of frequencies there will be two modes of surface wave propagation. Outside this range (at high or low frequencies) there will be just one mode. When both modes are possible, they seem to be of equal significance.

2. Basic equations. We quote the few equations from [1] that are needed subsequently, using the notation of that paper. A Rayleigh surface wave propagating over the free surface $x_{3}=0$ of a half-space of homogeneous, isotropic, incompressible, linearly-viscoelastic material will have a displacement $u_{i}$ of the form [1, Eq. (3.1)]

$$
u_{i}=\sum_{N=1}^{2} U_{i}^{(N)} \exp \left\{i \omega\left(s_{p}^{(N)} x_{p}-t\right)\right\},
$$

where $\omega$ is the real frequency of the wave. The slownesses $s_{i}^{(1)}, s_{i}^{(2)}$ satisfy [1, Eqs. (3.3), (3.4), (6.3)]

$$
\begin{aligned}
s_{1}{ }^{(1)} & =s_{1}^{(2)}=s_{1}, s_{2}{ }^{(1)}=s_{2}{ }^{(2)}=0, \\
{\left[s_{3}{ }^{\left({ }^{2}\right)}\right]^{2} } & =(\rho / \mu)-s_{1}{ }^{2}, s_{3}{ }^{(2)}=-i s_{1},
\end{aligned}
$$

where $x_{1}$ is the direction of propagation of the wave, $\rho$ is the constant density and $\mu$ is the complex Lamé modulus which is a function of frequency. If we write

$$
c=\rho / \mu s_{1}{ }^{2},
$$

* Received July 6, 1978.

** Present address: Koninklijke/Shell Exploratie en Produktie Lab., Postbus 60, Rijswijk, Netherlands. 
then $c$ is determined by the cubic secular equation derived from the boundary condition of zero traction on the top surface. The roots of this secular equation are [1, Eq. (6.2)],

$$
c_{1}=3.5437+2.2303 i, c_{2}=3.5437-2.2303 i, c_{3}=0.9126 .
$$

It is shown in [1] that $c_{1}$ is never an admissible root, since the disturbance corresponding to it does not decay with depth as required for a surface wave. If $\mu^{+}$and $\mu^{-}$are the real and imaginary parts respectively of $\mu$, then the root $c_{2}$ is admissible if [1, Eq. (6.8)]

$$
-\mu^{-} / \mu^{+}=\tan \delta>0.159
$$

where $\tan \delta$ is the loss tangent. This mode is called the viscoelastic mode (v.e.m.). It is not admissible if the material is purely elastic $\left(\mu^{-}=0\right)$. On the surface the elliptical particle path is described in the direct sense. For this mode [1, Eq. (6.5)]

$$
s_{3}{ }^{(1)}=-(1.7215-0.6478 i) s_{1} .
$$

The third root $c_{3}$ is always admissible. It is called the quasi-elastic mode (q.e.m.), since it is the only mode possible on a purely elastic material. On the surface the elliptical particle path is described in the retrograde sense. For this mode [1, Eq. (6.9)]

$$
s_{3}^{(1)}=-0.2956 i s_{1} \text {. }
$$

3. Standard linear solid. The constitutive equation for the stress $\sigma_{i j}$ at time $t$ in an incompressible standard linear solid is given by $[6$, p. 450]

$$
\sigma_{i j}=-p \delta_{i j}+2 G_{o}\left[e_{i j}(t)+\int_{-\infty}^{t}\left(\frac{1}{\tau}-\frac{1}{\theta}\right) \exp \left\{-\left(t-t^{\prime}\right) / \theta\right\} e_{i j}\left(t^{\prime}\right) d t^{\prime}\right]
$$

where $p$ is the arbitrary pressure and $e_{i j}$ the infinitesimal strain. $G_{0}$ is the elastic shear modulus, $\tau$ the characteristic time for creep and $\theta$ the characteristic time for relaxation. We note that $[6$, p. 451$]$

$$
\tau>\theta>0, G_{0}>0 .
$$

For this material, it follows that $\mu$ is given by [1, Eqs. (2.1)-(2.7)]

$$
\mu=G_{0}\left(\frac{\theta}{\tau}\right)\left(\frac{1-i \omega \tau}{1-i \omega \theta}\right) \text {. }
$$

Hence, from (2.5),

$$
\tan \delta=\omega(\tau-\theta) /\left(1+\omega^{2} \theta \tau\right) .
$$

The condition (2.5) is found to be satisfied if

$$
\omega_{1}<\omega<\omega_{2},
$$

where, using (3.2),

$$
\begin{aligned}
& \omega_{2}=\frac{1}{2 \tau}\left\{\left(\frac{k-1}{.159}\right)+\left[\left(\frac{k-1}{.159}\right)^{2}-4 k\right]^{1 / 2}\right\}, \\
& \omega_{1}=\frac{1}{2 \tau}\left\{\left(\frac{k-1}{.159}\right)-\left[\left(\frac{k-1}{.159}\right)^{2}-4 k\right]^{1 / 2}\right\}, \\
& k=\tau / \theta>1 .
\end{aligned}
$$

The critical frequencies $\omega_{1}$ and $\omega_{2}$ are complex if $k<1.37$. Thus for $1 \leq k \leq 1.37$ only the quasi-elastic mode is possible for the standard linear solid. For $k>1.37$ there is a range of 




FIG. 1. $k=2$. Variation of wave speeds and slownesses with frequency $\omega$. Quasi-elastic mode: (a) $v / V$, (b) $s_{1}{ }^{-} V$, (c) $s_{3}{ }^{-} V$. Viscoelastic mode: (â) $v / V$, (b) $s_{1}{ }^{-} V$, (c) $s_{3}{ }^{-} V$. Viscoelastic mode possible for $\ln \left|\omega / \omega^{*}\right|<1.44$.

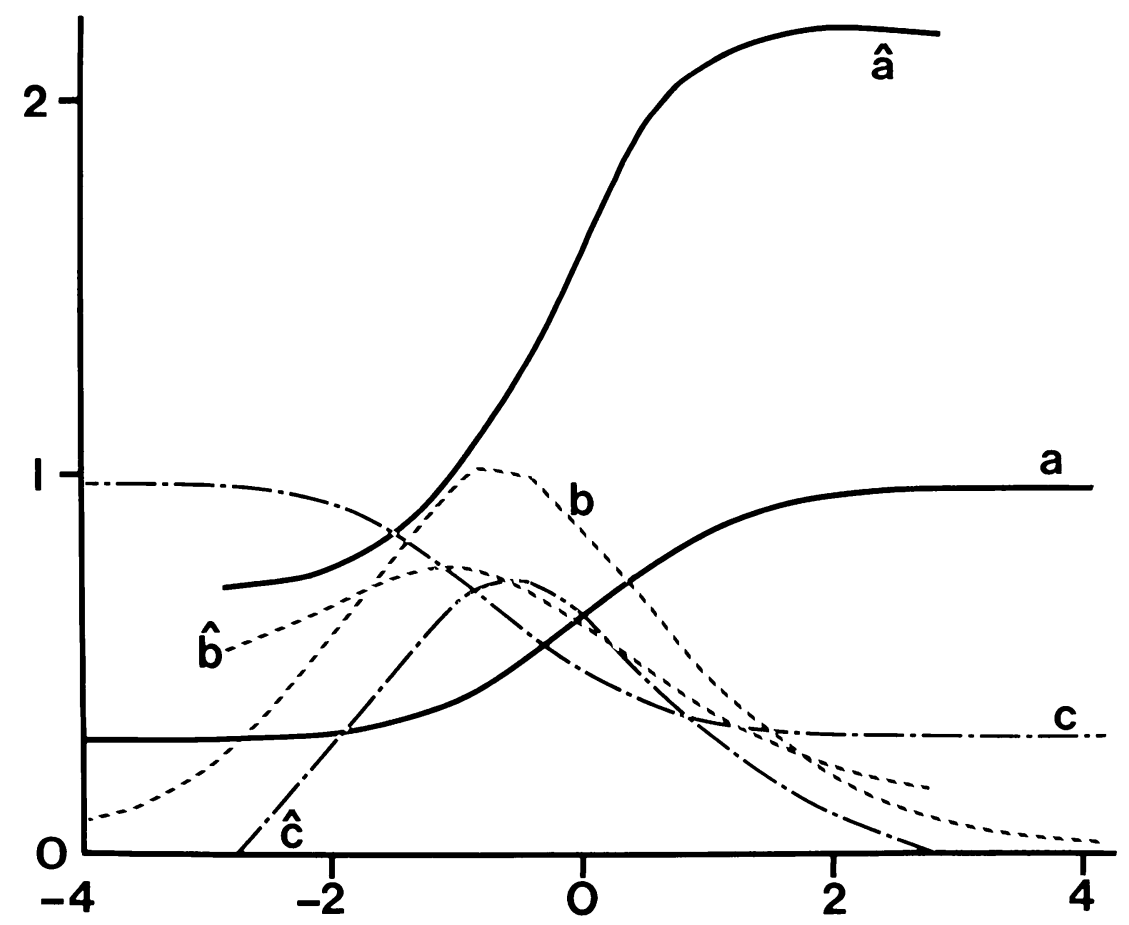

FIG. 2. $k=10$. As for Fig. 1. Viscoelastic mode possible for $\ln \left|\omega / \omega^{*}\right|<2.88$. 


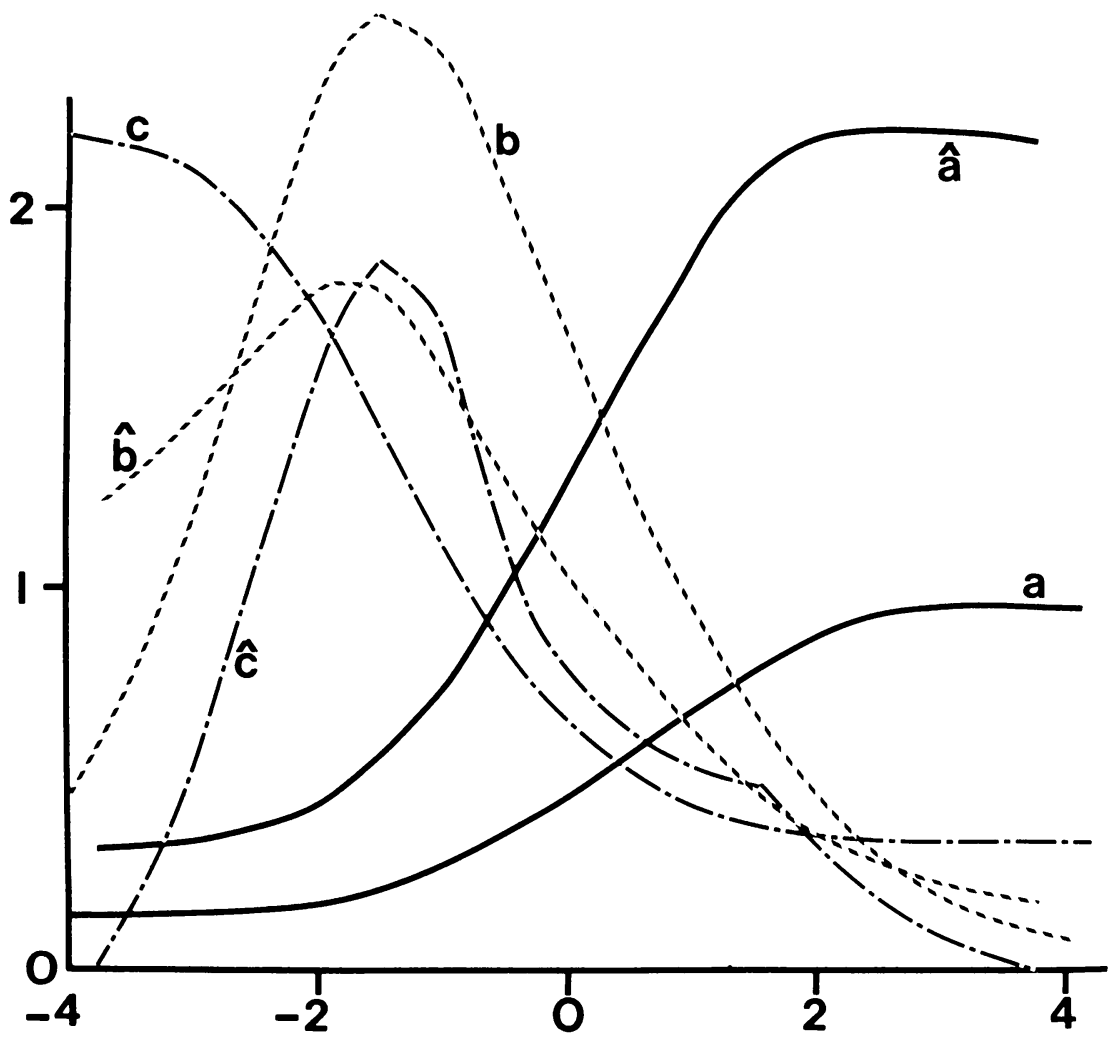

FIG. 3. $k=50$. As for Fig. 1. Viscoelastic mode possible for $\ln \left|\omega / \omega^{*}\right|<3.77$.

frequencies for which the solid has two surface modes.

Quantitites $\alpha, \beta, V$ are defined by

$$
\begin{aligned}
& \alpha=\left\{\frac{\tau}{\theta}\left[\frac{\left(\left[1+\omega^{2} \tau^{2}\right]\left[1+\omega^{2} \theta^{2}\right]\right)^{1 / 2}+1+\omega^{2} \theta \tau}{1+\omega^{2} \tau^{2}}\right]\right\}^{1 / 2},
\end{aligned}
$$

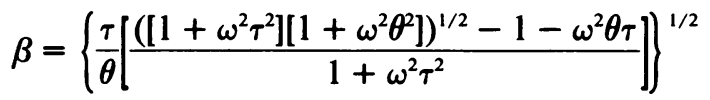

$$
\begin{aligned}
& V=\left(G_{0} / \rho\right)^{1 / 2} .
\end{aligned}
$$

$V$ is the body wave speed in an elastic material with shear modulus $G_{0}$. The following values of the slowness components are then found from (2.2)-(2.4).

Viscoelastic mode $c=c_{2}$ :

$$
\begin{aligned}
s_{1}{ }^{+}=s_{3}{ }^{(2)}{ }^{-} & =(0.332 \alpha-0.096 \beta) V^{-1}, \\
s_{1}{ }^{-} & =(0.096 \alpha+0.332 \beta) V^{-1}, \\
s_{3}{ }^{(1)} & =(0.050 \alpha-0.634 \beta) V^{-1} .
\end{aligned}
$$

Quasi-elastic mode $c=c_{3}$ :

$$
\begin{aligned}
s_{1}{ }^{+}=s_{3}{ }^{(2)^{-}} & =0.740 \alpha V^{-1}, \\
s_{1}- & =0.740 \beta V^{-1}, \\
s_{3}{ }^{(1)^{-}} & =-0.219 \alpha V^{-1} .
\end{aligned}
$$


We investigate these results by first taking $\omega=\omega^{*}$ where

$$
\omega^{*}=\left(\omega_{1} \omega_{2}\right)^{1 / 2}=(\theta \tau)^{-1 / 2} .
$$

$\omega^{*}$ is a typical value in the range of frequencies for which both modes are possible. We define the wave speed $v$ and the minimum slowness $s_{3}{ }^{-}$by

$$
v=1 / s_{1}{ }^{+}, s_{3}{ }^{-}=\min \left\{-s_{3}{ }^{(1)^{-}},-s_{3}{ }^{(2)}\right\} \text {. }
$$

Then it is found that at frequency $\omega^{*}$ :

(i) the speed $v$ over the top surface is greater for v.e.m. than for q.e.m.;

(ii) the exponential decay rate $\omega s_{1}^{-}$with distance along the top surface is less for v.e.m. than for q.e.m. if $k>2.61$;

(iii) the exponential rate of decay with depth $\omega_{3}{ }^{-}$is greater for v.e.m. than for q.e.m. if $k>6.13$.

Thus if $k>6.13$, then at the typical frequency $\omega^{*}$ the v.e.m. travels faster, suffers less attenuation with distance and decays more rapidly with depth than the q.e.m. It therefore seems to have the characteristics of a surface wave to a greater degree.

For further investigation we determine $k$ for materials in the transition zone from experimental values of the maximum of the loss tangent $\tan \delta$. From (3.4) this maximum value is $\frac{1}{2}\left(k^{1 / 2}-k^{-1 / 2}\right)$ and occurs at $\omega=\omega^{*}$. Comparison with Ferry [7, Fig. 12-9] gives the maximum of $\tan \delta$ as 1.5 for polyurethane rubber, 2 for poly-n-octyl methacrylate, 3.5 for polyvinyl acetate and 4 for unvulcanized Hevea rubber. The corresponding values of $k$ are 16, 23, 56 and 72. These choices of $k$ give curves for $\tan \delta$ as functions of $\omega$ which match the experimental curves reasonably well for polyurethane rubber and poly-n-octyl methacrylate, but for the other materials the curves rather overestimate $\tan \delta$ away from $\omega^{*}$.

In Figs. 1, 2, and 3 we plot the normalized wave speed $v / V$ and the slowness components $s_{1}{ }^{-} V$ and $s_{3}{ }^{-} V$ (governing decay along the surface and with depth) for both modes as functions of $\ln \left(\omega / \omega^{*}\right)$, taking $k=2,10,50$. It is seen that:

(i) when it is possible, the v.e.m. has greater wave speed than the q.e.m.;

(ii) for large $k(k \geq 10), s_{1}^{-}$is less for v.e.m. than for q.e.m. over at least the range $\left|\ln \left(\omega / \omega^{*}\right)\right| \leq 1.6$

(iii) for large $k(k \geq 10), s_{3}{ }^{-}$is greater for v.e.m. than for q.e.m. over at least the range $\left|\ln \left(\omega / \omega^{*}\right)\right| \leq 0.8$.

Thus, if it is accepted that this model is reasonable for the materials considered above, we can expect that over a range of about two decades of frequency centred on $\omega^{*}$ the v.e.m. will exist, and will travel faster, suffer less attenuation and decay more rapidly with depth than the q.e.m.

\section{REFERENCES}

[1] P. K. Currie, M. A. Hayes and P. M. O'Leary, Quart. Appl. Math. 35, 35 (1977).

[2] P. K. Currie and P. M. O'Leary, Quart. Appl. Math. 35, 445 (1978).

[3] P. K. Currie, Rheol. Acta, 17, 458 (1978).

[4] P. K. Currie, Mech. Res. Comm. 4, 403 (1977).

[5] H. A. Waterman, Rheol. Acta. 16, 31 (1977).

[6] S. C. Hunter, Mechanics of continuous media, Ellis Horwood (Wiley), Chichester, 1976

[7] J. D. Ferry, Viscoelastic properties of polymers, 2nd edition, Wiley, New York, 1970 